\title{
Cross Recruitment of Domain-Selective Cortical Representations Enables Flexible Semantic Knowledge
}

\author{
Scott L. Fairhall \\ Center for Mind/Brain Sciences, University of Trento, Rovereto, 38068, Italy
}

Knowledge about objects encompasses not only their prototypical features but also complex, atypical, semantic knowledge (e.g., "Pizza was invented in Naples"). This fMRI study of male and female human participants combines univariate and multivariate analyses to consider the cortical representation of this more complex semantic knowledge. Using the categories of food, people, and places, this study investigates whether access to spatially related geographic semantic knowledge (1) involves the same domain-selective neural representations involved in access to prototypical taste knowledge about food; and (2) elicits activation of neural representations classically linked to places when this geographic knowledge is accessed about food and people. In three experiments using word stimuli, domain-relevant and atypical conceptual access for the categories food, people, and places were assessed. Results uncover two principles of semantic representation: food-selective representations in the left insula continue to be recruited when prototypical taste knowledge is task-irrelevant and under conditions of high cognitive demand; access to geographic knowledge for food and people categories involves the additional recruitment of classically place-selective parahippocampal gyrus, retrosplenial complex, and transverse occipital sulcus. These findings underscore the importance of object category in the representation of a broad range of knowledge, while showing how the cross recruitment of specialized representations may endow the considerable flexibility of our complex semantic knowledge.

Key words: concepts; fMRI; food; MVPA; places

\section{Significance Statement}

We know not only stereotypical things about objects (an apple is round, graspable, edible) but can also flexibly combine typical and atypical features to form complex concepts (the metaphorical role an apple plays in Judeo-Christian belief). In this fMRI study, we observe that, when atypical geographic knowledge is accessed about food dishes, domain-selective sensorimotor-related cortical representations continue to be recruited, but that regions classically associated with place perception are additionally engaged. This interplay between categorically driven representations, linked to the object being accessed, and the flexible recruitment of semantic stores linked to the content being accessed, provides a potential mechanism for the broad representational repertoire of our semantic system.

Conceptual and semantic knowledge are critical, both for effective interaction with the environment and for higher thought. The complexity and flexibility of our semantic representational abilities make an understanding of its neural substrates a continuing challenge and an area of active research. Contemporary theories have coalesced around the notion that conceptual representation is accomplished through a combination of domain-

Received Sep. 11, 2019; revised Feb. 12, 2020; accepted Feb. 14, 2020.

Author contributions: S.L.F. designed research; S.L.F. analyzed data; S.L.F. wrote the paper.

This work was supported by European Research Council Grant CRASK (Cortical Representation of Abstract

Semantic Knowledge), under the European Union's Horizon 2020 research and innovation program (Grant Agreement 640594)

The author declares no competing financial interests.

Correspondence should be addressed to Scott L. Fairhall at Scott.fairhall@unitn.it.

https://doi.org/10.1523/JNEUROSCI.2224-19.2020

Copyright $\odot 2020$ the authors specific cortical representations, potentially linked through domain general representations unbound to specific sensorimotor associations (Damasio et al., 2004; Patterson et al., 2007; Mahon and Caramazza, 2008; Binder and Desai, 2011; Kiefer and Pulvermüller, 2012; Binder et al., 2016; Lambon-Ralph et al., 2017). Under these models, much of conceptual knowledge is acquired and contained within those neural populations active when we interact with the world and these conceptual representations are further bound into coherent generalizable concepts through a convergent hub or hubs.

Originating at its neuropsychological roots, inquiry has focused on object concepts and the role that semantic categories play in the cortical representation of these objects. Over decades, evidence has accumulated showing the importance of these categories and the probabilistic co-occurrences of features that delineate them: brain insult can selectively impair specific object 
categories (e.g., tools, animals, food) (for review, see Capitani et al., 2003, Rumiati and Foroni, 2016); brain regions are selectively involved in the representation of specific categories and related features (Noppeney et al., 2006; He et al., 2013; Fernandino et al., 2016; Martin, 2016); and in brain regions activated generally for semantic content (Binder et al., 2009), the voxel-level pattern of activation is sensitive to semantic object-category (Fairhall and Caramazza, 2013a).

While the investigation of the differential representation of object class in the brain has led to considerable advancements in our understanding, how we recognize and understand the typical aspects of objects represents a fraction of our rich semantic knowledge. Different kinds of knowledge about an object can be acquired and accessed in different ways. The shape, feel, color, and taste of an apple may be learned and represented through direct sensorimotor association. At the same time, it is hard to understand how snippets of knowledge, such as "all Granny Smith apples are propagate descendants of the same tree," could be similarly acquired through direct sensorimotor experience. Such knowledge is generally thought to be acquired "verbally," through symbolic language, and it is this verbally acquired conceptual knowledge that provides much of the depth and richness of human understanding, allowing us to combine basic elemental concepts into near-infinite combinations. Verbal knowledge differs from directly acquired knowledge in that its passage through domain-specific cortices is uninfluenced by semantic category, and it can more flexibly represent knowledge atypical to a category (e.g., Granny Smith apples come from Australia; they were discovered by Maria Ann Smith).

Category-selective representations continue to be recruited when accessed knowledge is unbound to sensorimotor experience. When equivalent information (e.g., nationality) is accessed about either people or places, distinct neural networks are recruited (Fairhall and Caramazza, 2013b; Fairhall et al., 2014). Moreover, there is some evidence for flexibility in this system. While accessing knowledge about specific famous people or places recruits only their respective category-selective representation, accessing knowledge about kinds of people or places, a baker or a bakery, results in an asymmetric cross-activation of the two systems. Accessing knowledge about types of people additionally recruits classically place-selective regions, implying that the representation of these concepts is distributed across person- and location-selective representational systems (Fairhall and Caramazza, 2013b).

The goal of the present study is to assess how atypical object knowledge is instantiated in the brain's semantic system. Specifically, (1) focusing on knowledge about traditional Italian food dishes, we consider whether atypical knowledge is represented in domain-selective cortical regions associated with sensorimotor representation; and (2) whether access to atypical knowledge (geographic region of origin) about the object categories food and people utilizes representations in brain regions traditionally associated with other object categories.

\section{Materials and Methods \\ Participants}

Twenty-six right-handed native Italian speakers were initially screened for participation in this study. As knowledge about provenance of items was anticipated to be variable in the population, only 20 participants who indicated they knew the provenance of at least $50 \%$ of each category's items completed the entire study. Four participants were excluded because head motion exceeded $3.0 \mathrm{~mm}$. Thus, we considered for the statistical analysis 16 participants (6 males, mean age 22 years).
Participants underwent a medical interview with a neurologist and did not present with history of neurologic disorder. All participants gave informed consent, and all procedures were approved by the University of Trento Human Research Ethics Committee.

\section{Experimental design}

Stimuli were 32 words taken from three semantic categories (96 in total) depicting traditional Italian dishes, famous Italian people, and Italian cities associated with a specific Italian region. The selected cultural feature, geographic knowledge in the form of the 13 (of 20) political/cultural regions of Italy from which the item originated, was chosen as this is unrelated to sensorimotor associations for food or people and broadly related to spatial information rather than a specific building or location. Additionally, 32 animal and tool words were used in the control condition.

The 32 stimuli in each category employed in the experiment were selected from an initial pool of 69 well-known food dishes, 115 famous people and 92 Italian cities. The final ratings for each included stimulus were based on the responses of 20 participants for 83 of the included items and 12 responses for the remaining 13 items. Matching was performed by removing items until the mean accuracy value was equivalent across categories. Matching was successful for people (73.7\%) and food (75.9\%) categories, but accuracies were significantly higher for cities $(85.8 \%, p$ values $<0.01)$. This is reflected in the behavioral responses for each category (see Results).

Because of the nature of the categories, word stimuli necessarily differed between categories in terms of number of letters (people, 13.9; food, 11.2; places, 6.6) and an associated within-category Levenshtein orthographic distance (people, 12.4; food, 10.9; places, 6.2). However, results distinguishing food from the other categories cannot be driven by this effect, as food stimuli are midway between places and people. All other comparisons considered the same stimuli across tasks.

The fMRI study consisted of three runs ( $10 \mathrm{~min}, 48 \mathrm{~s}$ each). Each run included 16 blocks, constituted of 8 word stimuli, belonging to one of the three semantic categories: food, person, city, and an additional control category composed of animal and tool names. Each block began with an $8000 \mathrm{~ms}$ colored fixation cross, the color of which indicated the semantic category of the upcoming block. The colored fixation cross was used to prime the participant for the upcoming judgment in the domainrelevant experiment and was maintained across experiments for reasons of consistency. Words were presented centrally in black for $500 \mathrm{~ms}$ and followed by $3500 \mathrm{~ms}$ of black fixation cross (total trial duration $4 \mathrm{~s}$ ). Both words and fixation crosses appeared on a grayscale phase-scrambled background. The presentation order of the blocks was pseudo-randomized. The stimuli were projected in the MRI scanner to a mirror above the subject's head. Responses were given on a two-button MR-compatible response device, using the left hand to dissociate the motor response interference from the left-lateralized semantic network. Psychophysics Toolbox Version 3 (www.psychtoolbox.org) and MATLAB (www.mathworks. com) were used to display the stimulation sequence.

Each of the three fMRI runs has a distinct experimental goal and task: domain-relevant access, provenance-retrieving, and provenance-matching. The domain-relevant access experiment was designed to elicit access to information relevant to each category (i.e., taste knowledge, social knowledge, spatial knowledge). In a one-back design, participants compared the presented item with the previous one along the domain-specific dimension, pressing one of two buttons to indicate whether the presented item was more or less tasty (food), nicer (people), or bigger (city) than the preceding one. The provenance-retrieving experiment was designed to elicit access to nonsensorimotor knowledge (i.e., Italian regional provenance). Participants indicated whether or not they knew the regional provenance of the presented item. The provenance-matching experiment likewise required access to the same geographic information but was cognitively demanding, leaving few resources available for processes unrelated to the task. Here, subjects performed a one-back matching task, where they consecutively indicated whether or not the presented item came from the same geographic region as the preceding one. Stimuli were pseudo-randomized to make the probability of a match equal to 0.2 . The control task was common across all experiments. 
Participants performed a one-back matching task indicating whether the presented nouns (tool/animal) were identical to the preceding one. This control task was designed to control for reading processes and motor response with minimal semantic processing.

The domain-relevant and provenance-matching experiments were counterbalanced in order, but provenance-retrieval was always run first to prevent the unnecessary priming of taste (or social) aspects of the stimuli. Additionally, this allowed us to exclude items where the provenance was unknown from the one-back tasks. Only items that were associated with a geographical origin in the provenance-retrieval run entered the other two experiments to ensure that judgments in subsequent tasks were performed on well-established information. The number of trials was kept fixed through repeated presentation of the same stimuli. Items were presented on average 1.71 times per experiment; and for each participant, the number of repetitions was balanced across the three object categories.

Before entering the scanner, participants were familiarized with the tasks by performing two abbreviated practice runs with a different set of stimuli.

\section{MRI scanning parameters}

Scanning was conducted on a Bruker BioSpin MedSpec 4T scanner and a USA Instruments eight-channel phased-array head coil. The experiment consisted of three runs of 328 echoplanar volumes. Each volume consisted of 34 slices aligned along the anterior/posterior commissure. The resulting image matrix was $70 \times 64$ voxels, with voxel size $3 \times 3 \times$ $3 \mathrm{~mm}$. TR was $2000 \mathrm{~ms}$, whereas TE was $33 \mathrm{~ms}$.

A T1-weighted MPRAGE structural image was acquired at the end of the experiment (voxel size $1 \mathrm{~mm}^{3}$, sagittal slice orientation, centric phase encoding, image matrix $256 \times 224$, FOV $256 \times 224 \mathrm{~mm}$, 176 slices with $1 \mathrm{~mm}$ thickness, GRAPPA acquisition with acceleration factor $=2$, duration $=5.36 \mathrm{~min}, \mathrm{TR}=2700, \mathrm{TE}=4.18, \mathrm{TI}=1020 \mathrm{~ms}, 7^{\circ}$ flip angle) .

\section{Postscanner test}

After the fMRI scans, subjects were asked to indicate the provenance of each item seen during the previous experiments. Participants were presented with each item accompanied by a list of the 13 possible Italian regions, and were instructed to choose one (forced choice).

\section{Statistical analyses}

fMRI data analyses were performed in SPM12 (https://www.fil.ion.ucl.ac. $\mathrm{uk} / \mathrm{spm} /$ ) and CoSMoVMPA (http://www.cosmomvpa.org) (Oosterhof et al., 2016) running on MATLAB (www.mathworks.com). After discarding the first four volumes of each run for residual T1 effects, all images were corrected for the slice-acquisition delay, using the middle slice as reference. EPI images were realigned to correct for head movement and normalized to the standard SPM12 MNI-EPI template. Volumes were resampled to a $3 \mathrm{~mm}$ isotropic voxel size. Images were spatially smoothed using an isotropic Gaussian kernel of $5 \mathrm{~mm}$ FWHM. For the multivariate analyses, data were high-pass filtered at 1/64 s.

A GLM was used to estimate the univariate neural response to the stimulation paradigm for each voxel, as a linear combination of effects of interest (stimuli, event duration $2 \mathrm{~s}$ ) and effect of no interest (six motion parameters) as well as regressors that affected a high-pass filter of $128 \mathrm{~s}$. For each subject, a statistical parametric map was computed for each condition. The resulting beta values were entered into a second level GLM and used to estimate a group-level random-effect response for each contrast of interest. Univariate contrasts were used to identify regional increases in the hemodynamic, and presumably neural, response. Weighted univariate contrasts were used as the primary analytical approach when a difference in response was hypothesized between conditions. This occurred in two contexts: (1) to identify whether a classical category-selective increase was seen for food stimuli presented as words in the domain-relevant task (contrast: [food > people and places]); and (2) to assess whether there was a greater response for food and people when atypical geographic information was accessed in the provenanceretrieval experiment than in the domain-relevant experiment (contrast: [food and people $>$ control $]_{\text {provenance-retrieval }}-[$ food and people $>$ control $]_{\text {domain-relevant}) \text {. }}$
ROI analyses were used to assess effects within a given region when motivated by theoretical or statistical considerations. To assess the influence of access to atypical geographic information about people and food items in classic place-selective regions, ROIs were constructed contrasting cities $>$ people and food in the domain-relevant experiment. This contrast identified six clusters, which corresponded to bilateral parahippocampal gyrus (PPA), transverse occipital sulcus (TOS), and retrosplenial complex (RSC) (compare Fig. 3B) at an uncorrected threshold $(p<$ 0.01 , extent $>15$ voxels). Place-selective regions are commonly localized with images and specific buildings or places, and the use of city stimuli depicted as words necessitated this relaxed threshold. Crucially, ROI definition is noninferential, and the interrogation of effects within these ROIs remains fully valid.

In a related follow-up analysis to assess the influence of intact geographic knowledge on these ROIs, trials where the object provenance was known were compared with trials where it was not in the provenance-retrieval experiment. New GLMs were formulated for each participant in the provenance-retrieval experiment. As in the original analysis, the response to each category was modeled but now with separate regressors for provenance "known" and "unknown" trials. This analysis was restricted to 12 participants who had unknown responses for each category. As the number of unknown trials were few and their distribution haphazard, Monte Carlo simulations were used to ensure the GLM design would not produce spurious differences between known and unknown trials. Parameters were identical to the previously described analysis preserving the frequency of known and unknown trials within each object category but randomly shuffling these "known" or "unknown" designations over 500 permutations. Specifically, new randomized GLMs were run for each subject and the results entered into a group-level GLM for ROI analyses (isolating bias due solely to the design characteristics). Paired $t$ test comparing known with unknown trials for each ROI and category and each permutation (9000 in total) yielded no significant increase in false positives.

Cross-experiment multivariate pattern analysis (MVPA) was used as the primary analytic approach to assess whether food-related information representation was consistent over differing forms of semantic access. This approach is optimal to address this question as it relies on information and representational states that are shared across experiments, is sensitive to subtle variations in the voxel-level patterns, and permits efficient usage of the full dataset in a single test (while a univariate conjunction analysis would necessitate three separate tests).

MVPA was performed on the echoplanar volumes corresponding to the near-asymptotic value of the convolved hemodynamic response function ( $\sim 6$ s delay; 52 volumes per condition) for each block. A searchlight classification procedure was implemented using a searchlight sphere of radius three voxels and linear discriminant analysis classification trained to discriminate between pairs of categories. Critically, linear discriminant analysis was trained on two experiments and tested on the remaining (leave-one out), for all the possible combinations (cross-validation), ensuring that only information consistent across experiments could inform the classifier. Accuracy maps were smoothed using a $6 \mathrm{~mm}$ FWHM isotropic Gaussian kernel. The presence of food-selective information was evaluated by first calculating voxels where there was more information distinguishing (1) food from people compared with people from places and (2) food from places compared with people from places. The resulting two maps were submitted to a random effect GLM for group-level inference ([food vs people + food vs places] $/ 2>$ [people vs places]; compare Fig. 2).

Two control ROI analyses were performed within an ROI defined on the maximally responsive section of the left insula identified in the domain-relevant food-selective contrast ( $p<0.05$, FWE voxel-corrected). This ROI approach was selected to maximize statistical power while avoiding any issue of circularity. First, the presence of univariate foodselective effects in the provenance access experiments that could account for any multivariate effects was assessed. Second, to ensure that any overall multivariate effects were not driven by food-selective representations common across only two of the three combinations of experiments, decoding accuracy across each pair of tasks was assessed and the searchlight summary voxels within this ROI averaged. Circularity in this 


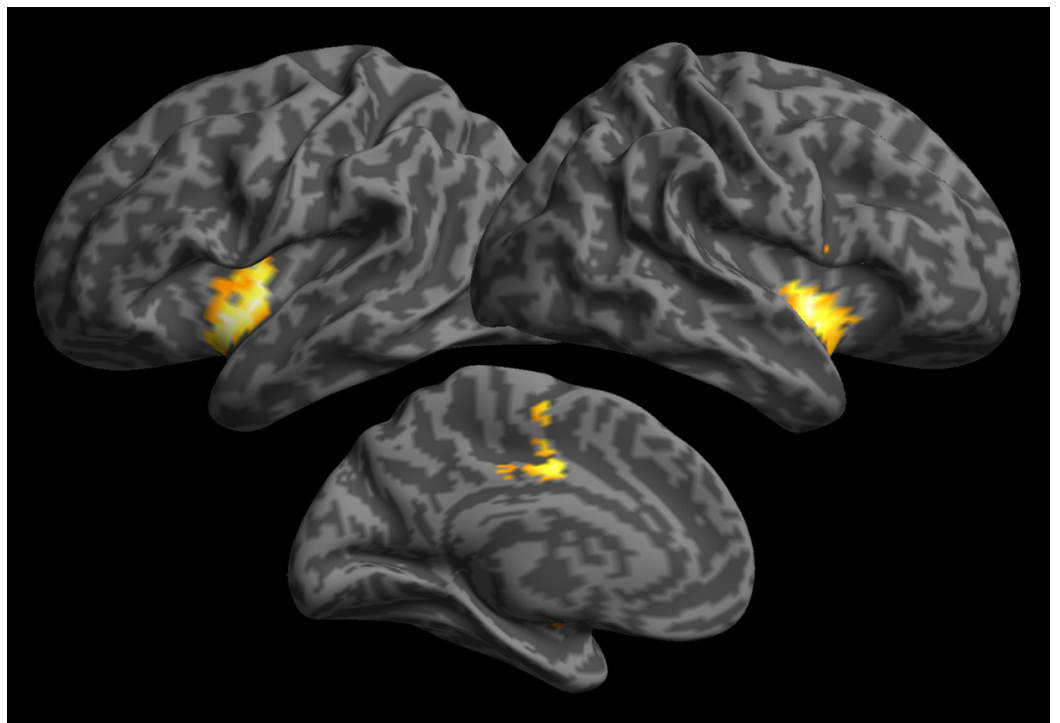

Figure 1. Word-cued food-selective activation when making taste judgments about famous Italian dishes. Shown is the univariate response comparing access with taste knowledge about food to domain-relevant judgments about people (likeability) and cities (size). Significant clusters of activation are present in bilateral insula and the left mid-cingulate gyrus ( $p<0.001$ voxelwise threshold, $p<0.05$ FWE cluster-corrected; see Table 1).

Table 1. Location, significance, and extent of clusters showing a greater domain-relevant response for food than for places and people

\begin{tabular}{lclllrrr}
\hline & Cluster P PWE & Voxels & Peak, $P_{\text {FWE }}$ & $t$ & \multicolumn{4}{c}{$x, y, z$ (MNI) } \\
\hline Left insula & $<0.001$ & 179 & 5.46 & 5.71 & -33 & -4 & 1 \\
Right insula & 0.001 & 147 & 4.30 & 5.26 & 36 & -1 & -5 \\
Left mid-cingulate gyrus & 0.007 & 116 & 4.70 & 4.82 & 0 & -7 & 43 \\
\hline
\end{tabular}

Table 2. Location, significance, and extent of clusters more active accessing the geographic domain-relevant content for food and people

\begin{tabular}{lllllrrr}
\hline & Cluster P PWE & Voxels & Peak, $P_{\text {FWE }}$ & $t$ & $x, y, z$ (MNI) & \\
\hline Left RSC & $<0.001$ & 352 & 0.001 & 5.71 & -6 & -61 & 13 \\
Right RSC & - & - & 0.011 & 5.26 & 9 & -49 & 4 \\
Left PPA & 0.023 & 62 & 0.061 & 4.82 & -24 & -37 & -17 \\
\hline
\end{tabular}

case is fully avoided as the data used to form the ROI only appear in either the testing or the training cross-validation folds.

\section{Results}

Behavioral

Participants' in-scanner subjective (yes/no) reports in the provenance-retrieval experiment $(69.1 \%$ people, $73.1 \%$ food, $87.1 \%$ place) matched closely to the objective (13 alternative forcedchoice) postscanner test (people $72.8 \%$, food $70.3 \%$, place $87.3 \%)$. While in-scanner reports of knowledge about people and food were comparable $\left(t_{(15)}<1\right)$, participants were more likely to know the region in which a city was located (people: $t_{(15)}=$ $2.76, p=0.015$; food: $\left.t_{(15)}=3.38, p=0.004\right)$. Response accuracy in the provenance-matching experiment was similar across semantic categories (people $86.33 \%$, food $85.29 \%$, place $85.85 \%$; $\left.F_{(2,45)}<1\right)$. Responses for the domain-relevant experiment were not analyzed due to their subjective nature.

Reaction times (RTs) did not differ between categories in Experiment 1 (food: 1159 ms; people: 1089 ms; places: 1282 ms; $p$ values $>0.38$ ), Experiment 2 (food: $1146 \mathrm{~ms}$; people: $1102 \mathrm{~ms}$; places: $989 \mathrm{~ms} ; \quad p$ values $>0.38$ ), or Experiment 3 (food: 1224 ms; people: $1189 \mathrm{~ms}$; places: $1003 \mathrm{~ms} ; p$ values $>0.16)$. Because of recording error, RT data were unavailable for 4 subjects in Experiment 1,6 in Experiment 2, and 3 in Experiment 3, and it is possible that undetected RT differences between places and the other two categories may be present. However, due to the inconsistency of RT differences across experiments and the similarity between food and people responses, these behavioral differences could not account for the fMRI results described hereafter.

\section{Word-cued access to taste knowledge selectively activates the insula}

To determine whether accessing domain-relevant taste knowledge about food selectively recruits particular brain regions, univariate analysis was used to identify food-selective increases in the fMRI response via the weighted contrast food $>$ [people and places] in the domain-relevant experiment. With respect to person and place stimuli, an increased response for food stimuli was seen in bilateral insulae, with a larger spatial extent in the left (Fig. 1; Tables 1, 2). We observed an additional food-selective increase in the middle cingulate gyrus.

\section{Common neural patterns for sensorimotor and verbal knowledge}

Next, we sought to determine whether the food-selective representations apparent when taste information is accessed are also active when (1) taste information is task-irrelevant and (2) the accessed information relates to nonsensory motor knowledge. To assess whether common representations are preserved across different types of semantic access, we turned to a cross-experiment MVPA. Cross-experiment MVPA was chosen as it allows the assessment of whether the representation of food evident in subtle voxel-level patterns of activity generalizes across forms of semantic access. Specifically, whether the differential pattern of brain responses produced by different categories observed in one retrieval context could be used to predict categorical responses in another retrieval context (for a similar approach with modality, see Fairhall and Caramazza, 2013a). For example, isolating that pattern of responses distinguishing food from people during the provenance-retrieving experiment and using this to predict the category producing neural activity in the domain-relevant experiment (Fig. 2). Explicitly, we ran a cross-experiment searchlight linear discriminant classifier that was trained on two experiments and tested on the remaining one. We repeated this analysis for each combination of category and experiment. In order to guarantee the food selectivity of the information, we identified regions in which the accuracy for food classification was greater than the accuracy for people and cities classification. Significantly greater classification accuracy demonstrates the presence of food-selected information, which is stable across experiments.

This whole-brain analysis revealed a pattern of representation of food knowledge that was consistent across tasks in the left insula (Fig. 2; Peak $\mathrm{MNI}_{\mathrm{MI}}$ : $36,-4,-2$, extent: 139 voxels, $p=$ 


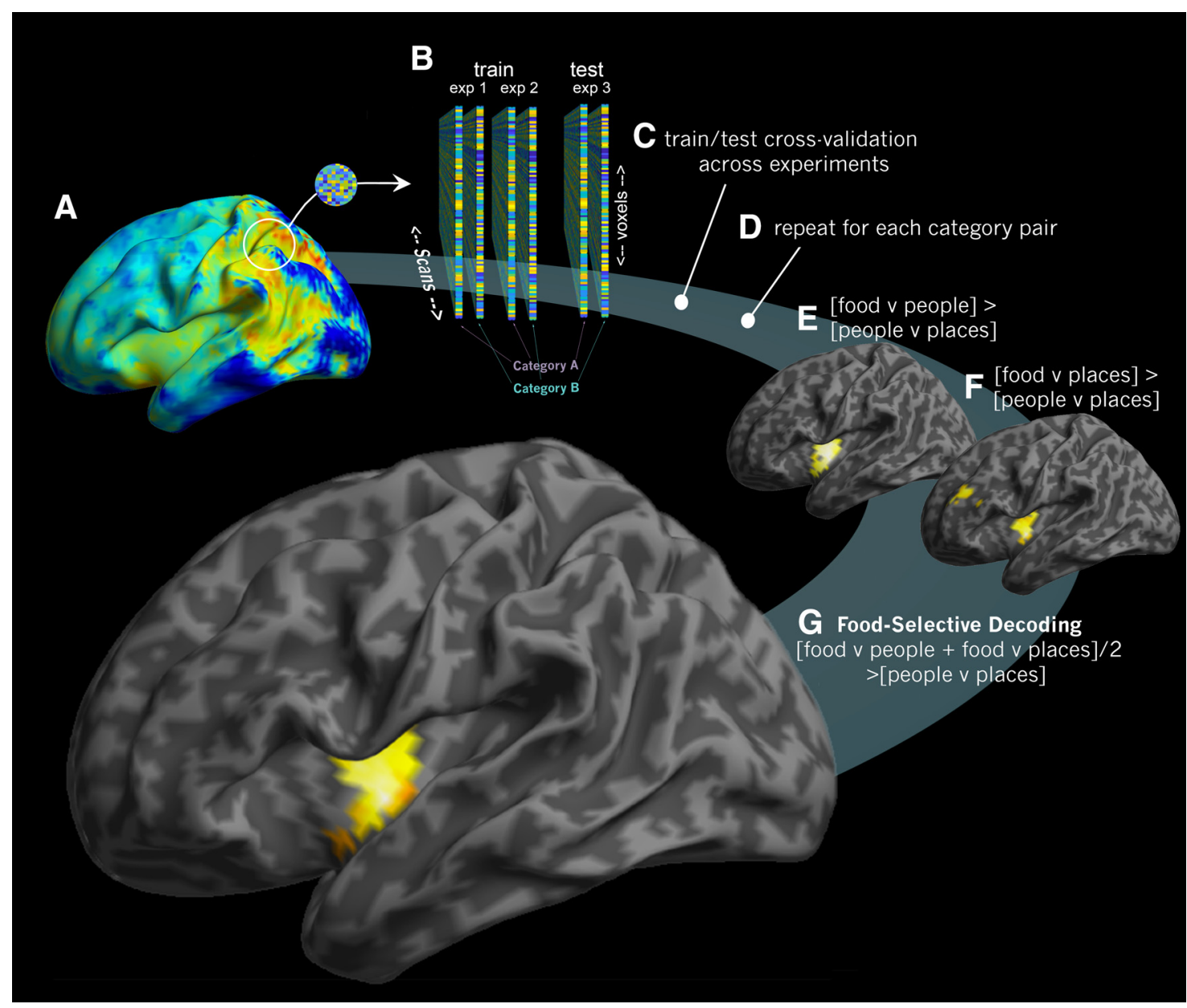

Figure 2. Food-selective representations that generalize across sensorimotor and verbal knowledge. $\boldsymbol{A}$, Searchlight linear discriminant analysis was performed by extracting the local patterns from the echoplanar images within a 3-voxel radius sphere consecutively centered at each cortical location. $\boldsymbol{B}$, Extracted patterns were then trained to distinguish between pairs of categories within two experiments and tested on the remaining experiment. C, This was performed in three cross-validation steps to isolate only information that was common across experiments. $\boldsymbol{D}$, This was repeated for the three category-pair combinations and brain maps formed of searchlight accuracies. Information content related to food was isolated by contrasting $\boldsymbol{E}$ ([food vs people] $>$ [people vs places]) and $\boldsymbol{F}$ ([food vs places] $>$ [people vs places]). $\boldsymbol{G}$, The resulting maps averaged for statistical inference via weighted contrast. Results reveal selective representations of food knowledge that are consistent across access to taste and geographic knowledge in the left insula $(p$ $<0.001$ voxelwise threshold, $p<0.05$ FWE cluster-corrected).

0.0168, corrected). The locus of this effect bared a marked similarity to the region identified in the domain-relevant response identified in the previous section. Inspection of the average decoding accuracy for each category pair within this region revealed comparable above-chance decoding accuracy for food versus people (3.1\%) and food versus places $(3.6 \%)$, which did not differ significantly from one another $(t<1)$. Decoding accuracy for people versus places was descriptively lower $(0.4 \%)$, but statistical comparison is invalid due to circularity.

A 47 voxel cluster in the right insula (peak MNI $_{36},-4,1$ ), did not survive multiple comparison corrections.

To assess whether subtle variations in the multivariate pattern were already evident in the overall fMRI response, univariate ROI analysis was performed on the left insula region defined in the domain-relevant experiment (Fig. 2). Results revealed no significant food selectivity (food $>$ [people and cities]) in the overall regional response for the provenance-retrieval $\left(t_{(15)}=1.2, p=\right.$ $0.23)$ or provenance-matching $\left(t_{(15)}=1.1, p=0.30\right)$ experiments, indicating that food-selective representations are present in the voxel-level pattern of the insula but not in the overall regional response.
To ensure that effects were not driven by patterns shared between only two of the three tasks, an alternate cross-validation strategy was implemented. For example, it is possible (if unlikely) that the left insula effect is driven by geographic information shared between provenance-retrieval and -matching experiments but not common to the domain-relevant experiment. Training and testing on pairs of experiments on data extracted from the left insula (defined by the univariate analyses in Fig. 1 to avoid circularity) revealed significant shared food information across experiments. Effects were strongest between provenance-retrieving and domain-relevant comparison experiments (above chance decoding accuracy: $7.0 \%, p<$ 0.001 ) and across provenance-matching and domain-relevant comparison (accuracy: $5.4 \%, p=0.002$ ) but also remained significant across the provenance-retrieving and the provenancematching experiments (accuracy: $3.3 \%, p<0.04$ ).

\section{Dual roles of object-domain and content-domain in semantic access}

When one accesses spatial information about food or people (regional provenance), are representations in place-selective regions recruited? Were this the case, one should observe the following: (1) 


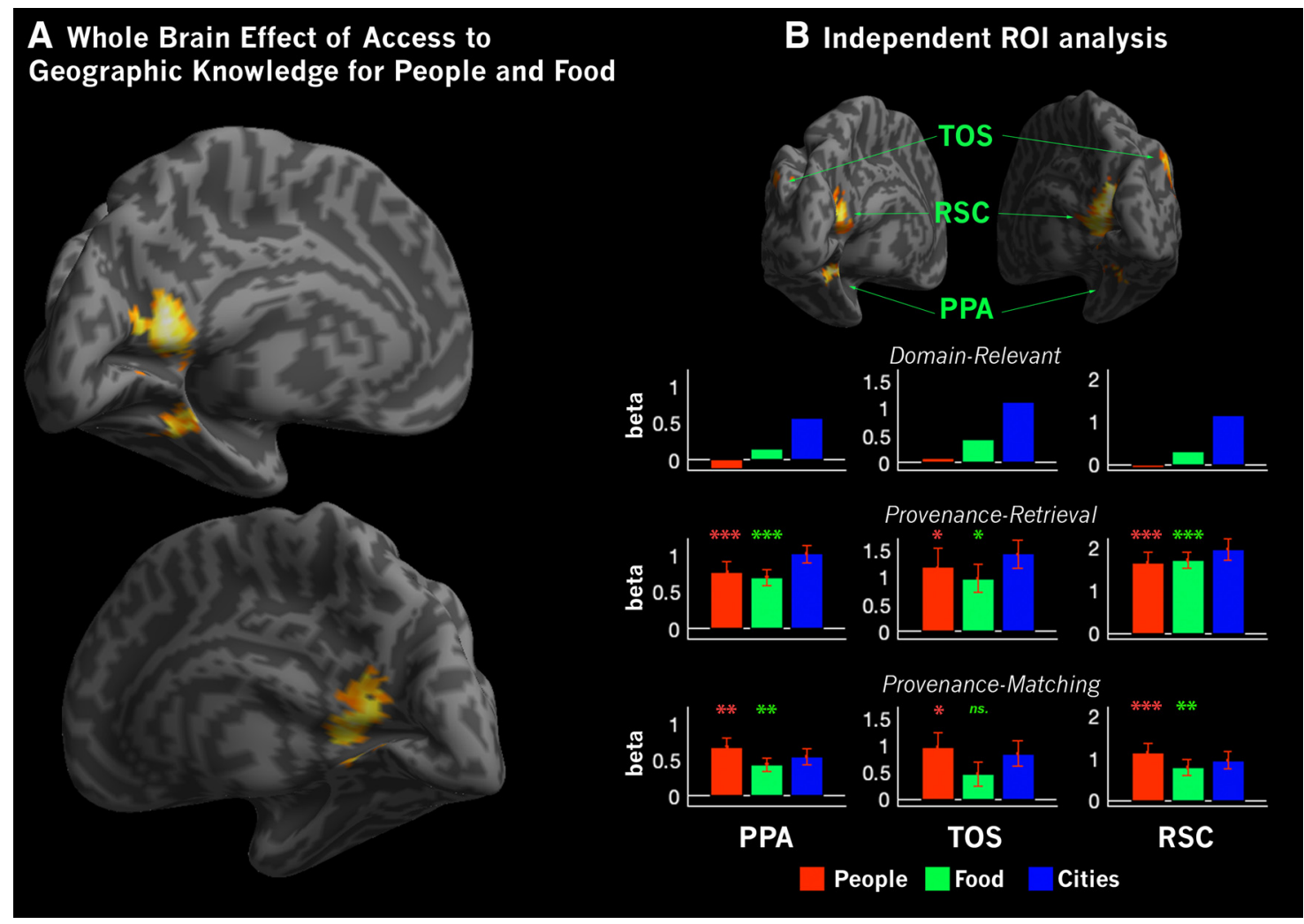

Figure 3. Selective effect of accessing geographic content for food and people compared with places. $\boldsymbol{A}$, Whole-brain analysis of increased cortical recruitment for access to geographic knowledge compared with domain-relevant content for food and people ( $p<0.001$ voxelwise threshold, ${ }^{*} p<0.05$ FWE cluster-corrected). $\boldsymbol{B}$, ROl analysis. ROls were defined on place-selective response in the domain-relevant experiment and interrogated in provenance-retrieval and -matching experiments. Signal plots show bilateral response in each ROI (error bar, \pm 1 standard error). Access to geographic knowledge increases responses for food and people in the place-selective network. Error bars are absent from the domain-relevant experiment plots due to the circularity of ROI definition/interrogation for this experiment. ${ }^{* * *} p<0.001,{ }^{* *} p<0.005,{ }^{*} p<0.05$.

significantly greater activation when access to geographic information is required than when it is not (i.e., social or gustatory); and (2) activation of place-selective regions during access to provenance information about people and food.

Univariate analyses were used to address whether betweencategory effects differed across experiments. A whole-brain analysis comparing greater responses for food and people during the provenance-retrieval task than the domain-relevant task (see Materials and Methods). This revealed significantly greater activation when geographic information was accessed in bilateral RSC and left PPA (Fig. 3A). ROI analysis was used to investigate this effect within classic place-selective regions. The contrast [cities] $>$ [food and people] in the domain-relevant experiment identified six regions that corresponded to bilateral PPA, TOS, and RSC. As an initial hemisphere $x$ region $\times$ experiment $\times$ task ANOVA revealed no interactions or main effects of hemisphere, subsequent analysis is reported collapsed across hemispheres. Planned paired $t$ tests revealed significant recruitment of the PPA (all $p$ values $<0.015$ ) and RSC (all $p$ values $<0.005$ ) by food and people categories when geographic information was accessed, compared with the control task. A similar pattern was seen in the TOS ( $p$ values $<0.05$ ), although effects were not evident for food items in the provenance-matching experiment. Thus, for both food and person stimuli, across the three ROIs and two experiments, in 11 of 12 cases, the results support crossregional processing of geographic knowledge.

To assess whether activation of place-selective regions during provenance-retrieval was contingent on knowledge of the provenance of the object, a follow-up analysis was performed comparing trials where the participants reported that the provenance was known with those where it was reported to be unknown (see Materials and Methods). This analysis showed a reduction in the response in place-selective ROIs when the provenance of a person or food exemplar was unknown. For people stimuli, this was apparent in PPA $\left(t_{(11)}=3.06\right.$, $p=0.005)$, TOS $\left(t_{(11)}=4.11, p<0.001\right)$, and RSC $\left(t_{(11)}=3.52\right.$, $p=0.002)$. On food-stimuli trials, this was evident in PPA $\left(t_{(11)}\right.$ $=2.17, p<0.026)$ and $\operatorname{RSC}\left(t_{(11)}=2.18, p=0.026\right)$. For city stimuli, no reduction in activity was evident when the regional location was unknown (all $t$ values $<1$ ). These results indicate that possession of geographical knowledge influences the response in place-selective regions for person and food stimuli.

\section{Discussion}

In this work, we address the interplay between category-selective semantic representations and the content of the knowledge being accessed. Specifically, we used the following: (1) MVPA to determine whether those food-selective representations apparent when sensorimotor-related information is accessed are also active when verbally weighted knowledge is accessed and taste knowledge is task-irrelevant; and (2) univariate analysis to determine whether the content of accessed knowledge increases activity within neural populations classically associated with other object categories related to that content. We found that a selective representation of food items in the insula, present when taste knowledge is accessed, continues to show a food-selective pattern of response when nonsensorimotor, verbally acquired geographical 
knowledge is accessed. Conversely, we observe that access to atypical geographical knowledge about food and famous people involves the cross recruitment of regions classically associated with knowledge about places.

\section{Word-cued category-selective activation for access to food knowledge}

A preliminary goal was to identify nonperceptual category-selective representations associated with food. The domain-relevant experiment revealed a food-selective response when taste information was accessed in bilateral insula, the probable location of primary gustatory cortices in humans (Small et al., 2003; Veldhuizen et al., 2011). The insula has been shown to be selectively activated when viewing images of food (Killgore et al., 2003; Simmons et al., 2005; for review, see van der Laan et al., 2011) and may reflect the automatic generation of inferences about taste when viewing images of food (Martin, 2016). Flavor perception is a multisensory experience combining taste, olfaction, and visual and auditory cues about food (Zampini and Spence, 2012). The present result shows that food-related insula activation is not solely the multisensory stimulus response to the image of food but persists during word reading. As the link between stimulus and gustatory response must pass through the abstract code of language, this finding indicates that conceptual representation contributes to the food-selective response in the insula.

Food-selective activation for access to taste knowledge was also seen in the mid-cingulate cortex. While remote from gustatory cortices, anatomic, resting-state, and functional coactivation data have shown this region to be tightly coupled with foodselective insula (Mesulam and Mufson, 1982; Taylor et al., 2009; Cauda et al., 2011), although the role of this region in access to taste information remains uncertain.

\section{Food-selective representations in the insula persist during taste-irrelevant semantic access}

Next, we consider whether domain-relevant representations continue to play a role when the associated feature (here, taste) is no longer relevant to the knowledge being accessed. Using a crossexperiment training/testing multivariate classifier, we determined whether food-selective patterns of response generalized across all three experiments, specifically, whether spatially related information, in the form of verbally weighted geographic knowledge, produced similar activation patterns to the retrieval of taste information. We observed that food-selective representations in the left insula continue to be recruited when taste information is task-irrelevant. The slow-paced domain-relevant task may encourage the incidental retrieval of non-task-relevant information, such as taste. In contrast, the high cognitive demand of the provenance-matching experiment, where presented items had to be matched with their preceding item in terms of the 13 possible geographic regions of origin, renders incidental retrieval unlikely. The generalization and preservation of the food-selective representation to all experiments argue that recruitment of representation in the insula is not epiphenomenal and is a part of WT semantic access. When we access information about an object unrelated to its category, domain-selective neural populations continue to add to the representation of that concept.

Collectively, these results show that food-selective semantic representations are present when accessing taste information and that these continue to play a role in representation across access to variations of semantic knowledge unrelated to sensorimotor food associations. This provides further support for the notion that conceptual representations may be grounded in sensorimotor experience (Kiefer and Pulvermüller, 2012; Martin, 2016) and that they are important for a broad range of semantic knowledge, extending beyond those primary sensorimotor associations to other, unrelated, forms of knowledge. The exact nature of this contribution, and its role in access to domainunrelated knowledge, remains a question for future research.

\section{Category and content interact to facilitate complex semantic knowledge}

Our final goal was to assess the interplay between the type of semantic content accessed about objects and cross-activation of category-selective semantic representations. Specifically, we addressed whether spatial information, in the form of verbally weighted geographic knowledge about famous Italian dishes and people, cross-recruited semantic representations normally associated with buildings and monuments. Whole-brain analysis revealed an increase in activation in bilateral RSC and left PPA for people and food when geographic information was accessed, compared with domain-relevant access. More sensitive ROI analysis confirmed this and showed that accessing geographic information about food and people increased responses across the network of place-selective brain regions, bilateral PPA, TOS, and RSC (Epstein and Kanwisher, 1998; Dilks et al., 2011; Persichetti and Dilks, 2018).

This network of classically place-selective regions has previously been shown to be recruited when we retrieve information about the nationality or vintage of famous monuments (Fairhall et al., 2014) and the function of various kinds of buildings (Fairhall and Caramazza, 2013a). Places, which are commonly and probabilistically associated with spatial knowledge, recruit these regions in the retrieval of a wide variety of information, even of a nonspatial nature (vintage, function). Here we see that other categories recruit this system when the specific kind of content being accessed is closely related to spatial information. This flexibility allows for both category-selective representations of information probabilistically associated with that category, while at the same time endowing the flexibility to recruit verbally acquired information related to the repertoire of information that can be flexibly accessed about the same object. As we saw, the recruitment of place-selective regions was stronger on trials where the province was known than when it was not. This relationship strengthens the link between spatially related geographic knowledge in the recruitment of these regions. However, it does not directly inform as to the nature of this representational content. Different potential mechanisms exist for the involvement of these regions in the atypical combination of geographic knowledge with people and food items. The conceptual system may consider broad geographic regions as a spatial feature associated with the object or as a separate discrete object, which is transiently linked to the person or food item. It is also possible that effects in place-selective regions arise only from the silent naming of the region. While this last alternative cannot be fully discounted, these place-selective regions are not typically associated with naming or language (Fedorenko and ThompsonSchill, 2014), and are strongly associated with spatially related cognition; and a semantic rather than nominal account for this effect remains the most parsimonious.

Inquiry into semantic representation is often focused on those fundamental, stereotypical, conceptual properties that allow us to directly interact with the world, the properties of knives, bread, and jam that allow us to make and consume a sandwich. At the same time, it is possible to ponder not just the taste or consistency of a food dish but its role in a particular historic event or the 
country or region from where it comes. It is these atypical aspects of objects, which may not arise automatically when presented with the item and do not represent features typically associated with that object class, that are critical to more elaborate semantic knowledge and higher-level thought. While models, such as the huband-spoke, posit that atypical indirect associations are made through a linking hub (Patterson et al., 2007), the mechanism behind the flexible conceptual combination of atypical semantic content associated with different domains remains largely unknown (Frankland and Greene, 2019). Collectively, the present results uncover two principles. First, category persistently drives representation, even when the accessed knowledge is unrelated to the defining features of that category. Second, complex knowledge about objects traverses category-selective cortices, with specific geographic content associated with food or people cross-recruiting those regions commonly associated with knowledge about places. This interplay between categorically driven representations, linked indelibly to the concept being accessed, and the flexible recruitment of semantic stores linked to the content being accessed, is a potential mechanism for the astounding functional repertoire of our semantic representational system. Future work will determine how these systems work with amodal nonselective representations reported previously (Binder et al., 2009; Fairhall and Caramazza, 2013a; Frankland and Greene, 2019) and are coordinated through semantic control regions (Thompson-Schill et al., 1997; Martin and Chao, 2001; Lambon-Ralph et al., 2017).

\section{References}

Binder JR, Conant LL, Humphries CJ, Fernandino L, Simons SB, Aguilar M, Desai RH, Binder JR, Conant LL, Humphries CJ, Simons SB, Aguilar M, Desai RH (2016) Toward a brain-based componential semantic representation. Cogn Neuropsychol 33:130-174.

Binder JR, Desai RH (2011) The neurobiology of semantic memory. Trends Cogn Sci 15:527-536.

Binder JR, Desai RH, Graves WW, Conant LL (2009) Where is the semantic system? A critical review and meta-analysis of 120 functional neuroimaging studies. Cereb Cortex 19:2767-2796.

Capitani E, Laiacona M, Mahon B, Caramazza A (2003) What are the facts of semantic category-specific deficits: a critical review of the clinical evidence. Cogn Neuropsychol 20:213-261.

Cauda F, D'Agata F, Sacco K, Duca S, Geminiani G, Vercelli A (2011) Functional connectivity of the insula in the resting brain. Neuroimage 55:8-23.

Damasio H, Tranel D, Grabowski T, Adolphs R, Damasio A (2004) Neural systems behind word and concept retrieval. Cognition 92:179-229.

Dilks DD, Julian JB, Kubilius J, Spelke ES, Kanwisher N (2011) Mirror-image sensitivity and invariance in object and scene processing pathways. J Neurosci 31:11305-11312.

Epstein R, Kanwisher N (1998) A cortical representation the local visual environment. Nature 392:598-601.

Fairhall SL, Caramazza A (2013a) Category-selective neural substrates for person- and place-related concepts. Cortex 49:2748-2757.

Fairhall SL, Caramazza A (2013b) Brain regions that represent amodal conceptual knowledge. J Neurosci 33:10552-10558.

Fairhall SL, Anzellotti S, Ubaldi S, Caramazza A (2014) Person- and placeselective neural substrates for entity-specific semantic access. Cereb Cortex 24:1687-1696.

Fedorenko E, Thompson-Schill SL (2014) Reworking the language network. Trends in cognitive sciences 18:120-126.
Fernandino L, Binder JR, Desai RH, Pendl SL, Humphries CJ, Gross WL, Conant LL, Seidenberg MS (2016) Concept representation reflects multimodal abstraction: a framework for embodied semantics. Cereb Cortex 26:2018-2034.

Frankland SM, Greene JD (2019) Concepts and compositionality: in search of the brain's language of thought. Annu Rev Psychol 71:273-303.

He C, Peelen MV, Han Z, Lin N, Caramazza A, Bi Y (2013) Selectivity for large nonmanipulable objects in scene-selective visual cortex does not require visual experience. Neuroimage 79:1-9.

Kiefer M, Pulvermüller F (2012) Conceptual representations in mind and brain: theoretical developments, current evidence and future directions. Cortex 48:805-825.

Killgore WD, Young AD, Femia LA, Bogorodzki P, Rogowska J, YurgelunTodd DA (2003) Cortical and limbic activation during viewing of highversus low-calorie foods. Neuroimage 19:1381-1394.

Lambon-Ralph MA, Jefferies E, Patterson K, Rogers TT (2017) The neural and computational bases of semantic cognition. Nat Rev Neurosci 18:42-55.

Mahon BZ, Caramazza A (2008) A critical look at the embodied cognition hypothesis and a new proposal for grounding conceptual content. J Physiol Paris 102:59-70.

Martin A (2016) GRAPES-grounding representations in action, perception, and emotion systems: how object properties and categories are represented in the human brain. Psychon Bull Rev 23:979-990.

Martin A, Chao LL (2001) Semantic memory and the brain: structure and processes. Curr Opin Neurobiol 11:194-201.

Mesulam MM, Mufson EJ (1982) Insula of the Old World monkey: architectonics in the insulo-orbito-temporal component of the paralimbic brain. J Comp Neurol 212:1-22.

Noppeney U, Price CJ, Penny WD, Friston KJ (2006) Two distinct neural mechanisms for category-selective responses. Cereb Cortex 16:437-445.

Oosterhof NN, Connolly AC, Haxby JV (2016) CoSMoMVPA: multi-modal multivariate pattern analysis of neuroimaging data in MATLAB/GNU octave. Front Neuroinform 10:27.

Patterson K, Nestor PJ, Rogers TT (2007) Where do you know what you know? The representation of semantic knowledge in the human brain. Nat Rev Neurosci 8:976-987.

Persichetti AS, Dilks DD (2018) Dissociable neural systems for recognizing places and navigating through them. J Neurosci 38:10295-10304.

Rumiati RI, Foroni F (2016) We are what we eat: how food is represented in our mind/brain. Psychon Bull Rev 23:1043-1054.

Simmons WK, Martin A, Barsalou LW (2005) Pictures of appetizing foods activate gustatory cortices for taste and reward. Cereb Cortex 15:16021608.

Small DM, Gregory MD, Mak YE, Gitelman D, Mesulam MM, Parrish T (2003) Dissociation of neural representation of intensity and affective valuation in human gustation. Neuron 39:701-711.

Taylor KS, Seminowicz DA, Davis KD (2009) Two systems of resting state connectivity between the insula and cingulate cortex. Hum Brain Mapp 30:2731-2745.

Thompson-Schill SL, D'Esposito M, Aguirre GK, Farah MJ (1997) Role of left inferior prefrontal cortex in retrieval of semantic knowledge: a reevaluation. Proc Natl Acad Sci USA 94:14792-14797.

van der Laan LN, de Ridder DT, Viergever MA, Smeets PA (2011) The first taste is always with the eyes: a meta-analysis on the neural correlates of processing visual food cues. Neuroimage 55:296-303.

Veldhuizen MG, Albrecht J, Zelano C, Boesveldt S, Breslin P, Lundström JN (2011) Identification of human gustatory cortex by activation likelihood estimation. Hum Brain Mapp 32:2256-2266.

Zampini M, Spence C (2012) Assessing the role of visual and auditory cues in multisensory perception of flavor. The neural bases of multisensory processes. Boca Raton, FL: CRC/Taylor and Francis. 